\title{
Normal Map Acquisition of Nearly Flat Objects Using a Flatbed
}

\author{
Scanner \\ Rongjiang Pan ${ }^{1}$, Vaclav Skala ${ }^{2}$ \\ ${ }^{1}$ School of Computer Science and Technology, Shandong University, Jinan, China, \\ panrj@sdu.edu.cn \\ ${ }^{2}$ Faculty of Applied Sciences, University of West Bohemia, Plzen, Czech Republic \\ skala@kiv.zcu.cz
}

\begin{abstract}
We propose a new efficient technique for acquiring normal maps of nearly flat objects using a flatbed scanner. The technique requires no hardware modification or dedicated calibration. Our setup consists of a conventional flatbed scanner and a clamp on which a planar checkerboard is glued. We take four scans of the object manually rotated by roughly 90 degrees. The scans are then registered by subpixel checkerboard corners. Detailed normal map of the scanned surface is obtained by solving a linear least squares problem.
\end{abstract}

Keywords normal map reconstruction, subpixel corners, flatbed scanner, checkerboard

\section{Introduction}

Many small antiques such as seals and coins have a nearly flat surface as shown in Figure 1. The surface details, including scratches and impressions, are important information for archaeological study. However, existing range scanners have difficulties in capturing such subtle details on the surface due to their low resolution. Although microgeometry capture systems using elastomeric sensor are proposed recently [1][2], they are still in prototype stage and require special sensor materials. Images with normal maps, which contain a color and a surface normal channel for each pixel, are a type of data lying between simple 2D images and full 3D models. In contrast to photographs and 3D models, normal maps make sharp changes in normal direction clearly visible [3]. Images with normal vectors can also be used to produce detailed and understandable illustrations of complex real-world objects [4].

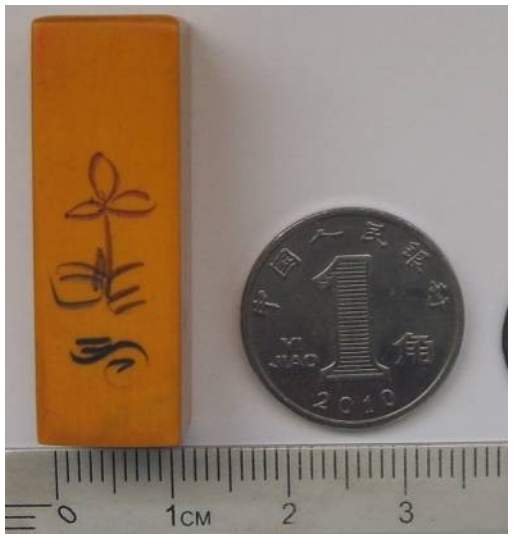

Figure 1: Two small objects which have a nearly flat surface

In this paper we introduce a method to acquire normal maps of nearly flat objects using a flatbed scanner. Nowadays flatbed scanners are low cost and mature products to digitize documents and photographs at high resolution. The hardware setup consists of a conventional flatbed scanner and a clamp on which a planar checkerboard pattern is glued. Our contributions 
include:

- A normal maps acquisition system that requires no hardware modification or dedicated calibration.

- A robust algorithm for registering multiple scans by virtue of subpixel checkerboard corners.

- Easily controlled and accurate lighting directions for four scans rotated by 90 degrees with a clamp.

\section{Related Work}

Normal maps are widely used in real-time rendering [5] and geometric modeling [6]. It is a technique used for faking the lighting of bumps and dents, adding details without using more polygons. Normal maps can be extracted from a high resolution modeled mesh and enhance the appearance and details of a low polygon model.

For real world objects, normal maps are often reconstructed from multiple images under varying illumination based on the principle of photometric stereo [7]. Digital cameras can be used to take multiple images from the same view point illuminated with different light sources of known position. Under the assumptions of perfect diffuse shading, per-pixel surface normals of an object can also be obtained from multiple scans at different orientations with a flatbed scanner. Brown et al. [3] obtained a normal map of a fresco fragment from four scans rotated by approximately 90 degrees with a flatbed scanner. The scanned images are registered via an affine transform using a normalized cross-correlation. They also required a dedicated calibration phase using a diffuse plane inclined at known angles with respect to the scanner platform. Clarkson et al applied photometric stereo to four scans of the paper using a commodity flatbed scanner [9]. They used a printed box on the test pages to align different scans and recovered each surface normal's projection into xy-plane up to a scale, $\left(n_{\mathrm{x}}, n_{\mathrm{y}}\right)^{T}$. Pintus et al extracted normals and albedo of objects using a two-bulb flatbed scanner [8]. They activated each of the bulbs separately by modifying scanner hardware or using private software unavailable to others. They developed a registration method by simultaneous registration and photometric stereo. However, chromatic difference between bulbs and imperfect mechanical repeatability of the scanner are problematic for photometric stereo. They modeled the scanner's light as point light sources. Instead, flatbed scanners contain a linear light source, which is placed at a fixed angle with respect to the moving CCD [3] [8].

Our work is an improvement over Brown's [3] and Clarkson's [9] methods. Compared with Brown's method, our method does not require calibration procedure and is more adaptable. In contrast to Clarkson's method, we recover the normalized 3D surface normals, $\left(n_{\mathrm{x}}, n_{\mathrm{y}}, n_{\mathrm{z}}\right)^{T}$. As will be explained in next section, it has an important effect on the extracted normals to rotate each scan by approximately 90 degrees. To obtain four scans rotated by 90 degrees as accurate as possible, we make use of a clamp which can be easily placed along the side of the scanner platen.

\section{Acquisition Workflow}

Photometric stereo usually requires at least three images of the surface to estimate surface normals [10]. Typically, more than the minimum three images are used, providing noise reduction. To make it easier and robust, we take scans at orientations $0^{\circ}, 90^{\circ}, 180^{\circ}$ and $270^{\circ}$ although 
other orientations could be used as well. In order to make the position of the object as accurate as possible within the checkerboard, we utilize a clamp from numerical control machines (Figure 2) to fix the scanned object. A planar checkerboard pattern is glued on the clamp and utilized in the multiple scans registration. However, other fixing systems that keep the object collinear with the scanner scanning surface can be used as well.

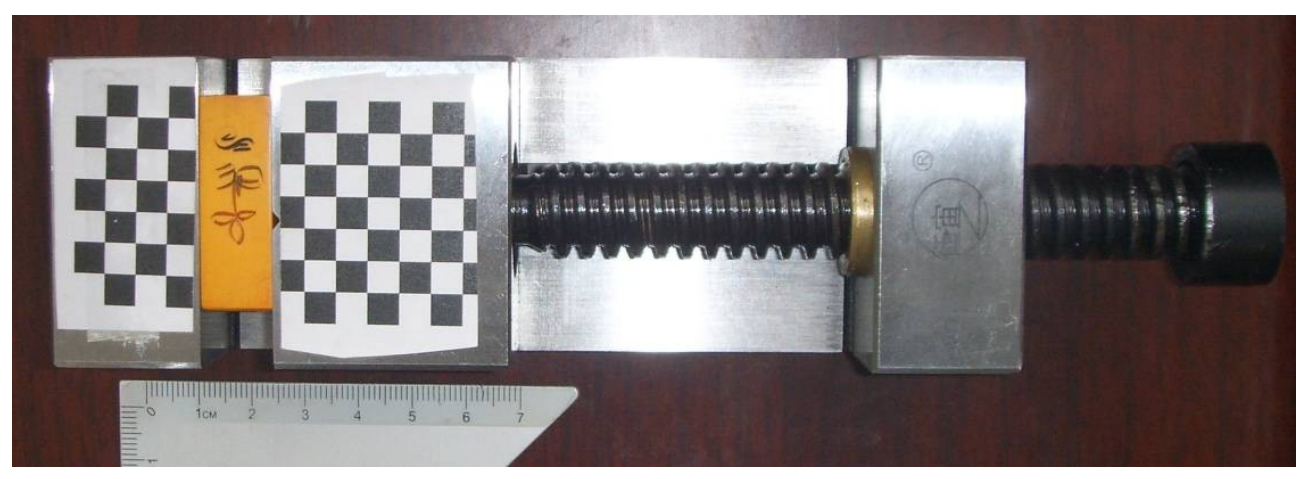

Figure 2: A clamp to fix the scanned object

\subsection{Multiple Scans Registration}

We make use of checkerboard corners to register multiple scans. A custom-designed user interface was provided for a semi-automated subpixel corner extraction. At least three corners are selected on the rectangular checkerboard pattern. Afterwards, subpixel detection techniques are applied to corners for refining their locations to subpixel accuracy [11].

Assume that in two scans there exist corresponding corners $\left\{\mathbf{x}_{i}\right\}$ and $\left\{\mathbf{y}_{i}\right\}, i=1, \cdots$, such that they are related by:

$$
\mathbf{x}_{i}=\mathbf{R y}_{i}+\mathbf{t}
$$

where $\mathbf{R}$ is a standard $2 \times 2$ rotation matrix and $\mathbf{t}$ is a $2 \mathrm{D}$ translation vector. Solving for the optimal transformation typically requires minimizing

$$
S^{2}=\sum_{i=1}^{n}\left\|\mathbf{x}_{i}-\mathbf{R} \mathbf{y}_{i}-\mathbf{t}\right\|^{2}
$$

The two sets of corners $\left\{\mathbf{x}_{i}\right\}$ and $\left\{\mathbf{y}_{i}\right\}$ should have the same centroid. Defining $\overline{\mathbf{x}}=\sum_{i=1}^{n} \mathbf{x}_{i}, \mathbf{x}_{i}^{\prime}=\mathbf{x}_{i}-\overline{\mathbf{x}}$ and $\overline{\mathbf{y}}=\sum_{i=1}^{n} \mathbf{y}_{i}, \mathbf{y}_{i}^{\prime}=\mathbf{y}_{i}-\overline{\mathbf{y}}, \quad$ the Eq. (2) can be reduced to

$$
S^{2}=\sum_{i=1}^{n}\left\|\mathbf{x}_{i}^{\prime}-\mathbf{R} \mathbf{y}_{i}^{\prime}\right\|^{2}=\|\mathbf{X}-\mathbf{R Y}\|_{F}^{2}
$$

where $\mathbf{X}$ and $\mathbf{Y}$ are matrices whose columns are formed from the corner position vectors, $\|\cdot\|_{F}$ is the Frobenius norm.

Minimizing $\|\mathbf{X}-\mathbf{R Y}\|_{F}^{2}$ is equivalent to maximizing Trace $\left(\left(\mathbf{Y} \mathbf{X}^{T}\right) \mathbf{R}\right)$. According to the Lemma 1 in [12], the optimal rotation matrix is

$$
\mathbf{R}=\left[\begin{array}{cc}
1 & 0 \\
0 & \operatorname{det}\left(\mathbf{U} \mathbf{V}^{T}\right)
\end{array}\right]
$$

and the translation vector is $\mathbf{t}=\overline{\mathbf{x}}-\mathbf{R} \overline{\mathbf{y}}$ where $\mathbf{U S V ^ { T }}$ is the Singular Value Decomposition 
(SVD) of $\mathbf{X Y} \mathbf{Y}^{T}, \mathbf{U}$ and $\mathbf{V}$ are orthogonal matrices, $\mathbf{S}$ is a diagonal matrix.

After aligning the other three scans to the first scan, we cut out the image regions that are out of interest.

\subsection{Normal Reconstruction}

The light source of a flatbed scanner is linear and placed at a fixed angle $\alpha$ with respect to the scanner platen. Each point on the scanned surface has a normal $\mathbf{n}$ and albedo $\rho$. For the scanning taken at orientation $0^{\circ}$, we define a left-handed coordinate system as shown in Figure 3(a) whose origin is at the surface point considered, $x$-axis is parallel to the scanner's linear light source, xy-plane is the scanner platen and z-axis points down. The light source is approximated by a line segment extending from $-l$ to $+l$ in the $\mathrm{x}$-axis direction and is offset by $a$ in the $\mathrm{y}$-direction, $b$ in the z-direction. We define $\mathbf{n}=\left(n_{\mathrm{x}}, n_{\mathrm{y}}, n_{\mathrm{z}}\right)^{T}$ as a normalized surface normal at the point, $\mathbf{I}=\frac{(x, a, b)^{T}}{\sqrt{x^{2}+a^{2}+b^{2}}}$ is the normalized lighting direction vector along the linear light source. The observed intensity of such a surface point is then:

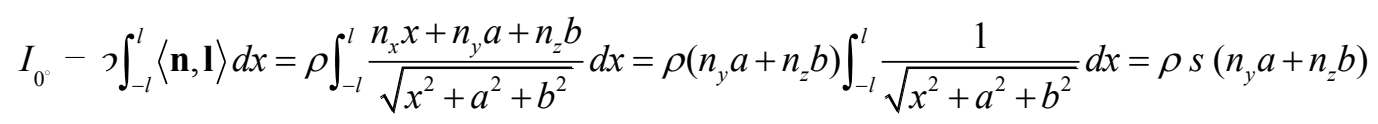

where $s=\int_{-l}^{l} \frac{1}{\sqrt{x^{2}+a^{2}+b^{2}}} d x=2 \ln \frac{\left(l+\sqrt{l^{2}+a^{2}+b^{2}}\right)}{\sqrt{a^{2}+b^{2}}}$.

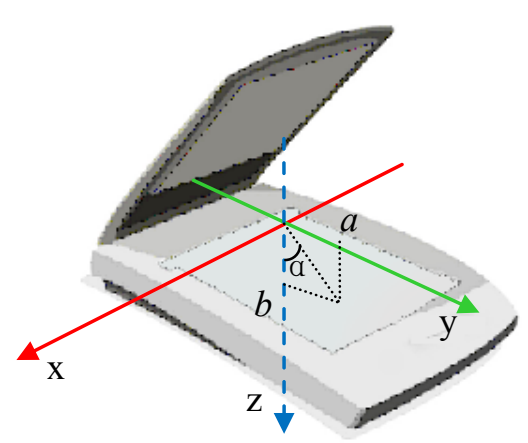

(a) $0^{\circ}$

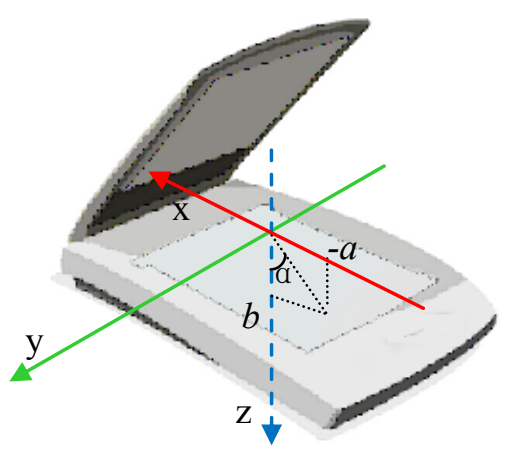

(c) 90

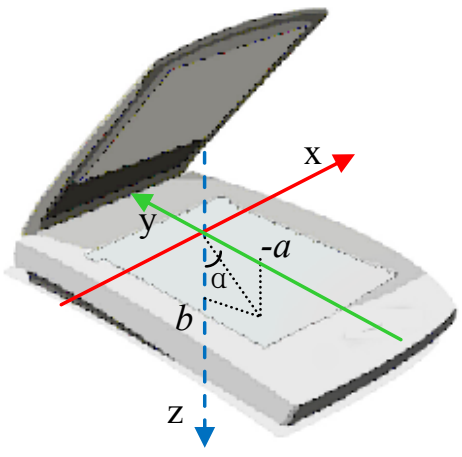

(b) $180^{\circ}$

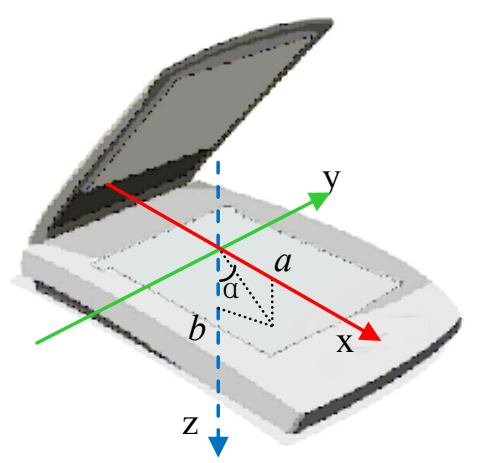

(d) 270

Figure 3: Four scanning taken at different orientations 
Analogously, scanning the same surface point with the object rotated by $180^{\circ}$ whose normalized lighting direction vector along the linear light source is $\mathbf{I}=\frac{(x,-a, b)^{T}}{\sqrt{x^{2}+a^{2}+b^{2}}}$ as shown in Figure 3(b) leads to:

$$
I_{180^{-}}-\jmath \int_{-l}^{l}\langle\mathbf{n}, \mathbf{l}\rangle d x=\rho \int_{-l}^{l} \frac{n_{x} x-n_{y} a+n_{z} b}{\sqrt{x^{2}+a^{2}+b^{2}}} d x=\rho\left(-n_{y} a+n_{z} b\right) \int_{-l}^{l} \frac{1}{\sqrt{x^{2}+a^{2}+b^{2}}} d x=\rho s\left(-n_{y} a+n_{z} b\right)
$$

Scanning the same surface point with the object rotated by $90^{\circ}$ whose normalized lighting direction vector along the linear light source is $\mathbf{l}=\frac{(-a, y, b)^{T}}{\sqrt{a^{2}+y^{2}+b^{2}}}$ as shown in Figure 3(c) leads to:

$$
I_{90^{\circ}}-\iint_{-l}^{l}\langle\mathbf{n}, \mathbf{I}\rangle d y=\rho \int_{-l}^{l} \frac{-n_{x} a+n_{y} y+n_{z} b}{\sqrt{a^{2}+y^{2}+b^{2}}} d y=\rho\left(-n_{x} a+n_{z} b\right) \int_{-l}^{l} \frac{1}{\sqrt{a^{2}+y^{2}+b^{2}}} d y=\rho s\left(-n_{x} a+n_{z} b\right)
$$

Scanning the same surface point with the object rotated by $270^{\circ}$ whose normalized lighting direction vector along the linear light source is $\mathbf{I}=\frac{(a, y, b)^{T}}{\sqrt{a^{2}+y^{2}+b^{2}}}$ as shown in Figure 3(d) leads to:

$$
I_{270^{-}}-\vartheta \int_{-l}^{l}\langle\mathbf{n}, \mathbf{I}\rangle d y=\rho \int_{-l}^{l} \frac{n_{x} a+n_{y} y+n_{z} b}{\sqrt{a^{2}+y^{2}+b^{2}}} d y=\rho\left(n_{x} a+n_{z} b\right) \int_{-l}^{l} \frac{1}{\sqrt{a^{2}+y^{2}+b^{2}}} d y=\rho s\left(n_{x} a+n_{z} b\right)
$$

We may arrange the four equations into a matrix equation:

$$
\left[\begin{array}{ccc}
0 & \tan \alpha & 1 \\
0 & -\tan \alpha & 1 \\
-\tan \alpha & 0 & 1 \\
\tan \alpha & 0 & 1
\end{array}\right]\left[\begin{array}{l}
\rho s b n_{x} \\
\rho s b n_{y} \\
\rho s b n_{z}
\end{array}\right]=\left[\begin{array}{cc}
I_{0^{\circ}} & \text { । } \\
I_{180^{\circ}} & \mid \\
I_{90^{\circ}} & \mid \\
I_{270^{\circ}} &
\end{array}\right.
$$

where $\tan \alpha=\frac{a}{b}$. Equation 4 can be solved using linear least-squares. Its solution is

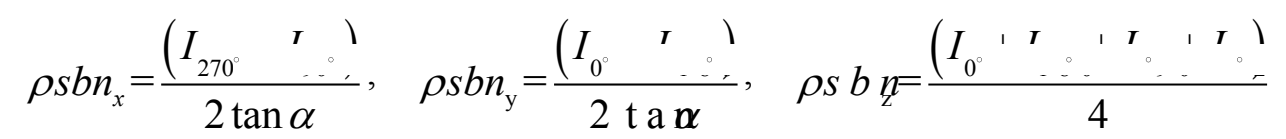

According to [8], the lighting geometry is approximated with $\alpha=\pi / 6$. Finally, the normal $\mathbf{n}$ at this surface point is obtained and normalized.

\subsection{Mean Curvature Estimation}

Once we have obtained normal vectors at every surface point, we estimate the curvatures of the surface similarly to [4]. Assuming the surface has the explicit form $z=z(x, y)$, we first transform the normal $\mathbf{n}=\left(n_{\mathrm{x}}, n_{\mathrm{y}}, n_{\mathrm{z}}\right)^{T}$ into $\mathbf{n}=\left(n_{\mathrm{x}}, n_{\mathrm{z}}, n_{\mathrm{y}} / n_{\mathrm{z}}, 1\right)^{T}$. The mean curvature is half the trace of 
$\mathrm{I}^{-1} \mathrm{II}$ where $\mathrm{I}$ is the first fundamental tensor

$$
\mathrm{I}=\left(\begin{array}{cc}
\mathbf{u} \cdot \mathbf{u} & \mathbf{v} \cdot \mathbf{u} \\
\mathbf{u} \cdot \mathbf{v} & \mathbf{v} \cdot \mathbf{v}
\end{array}\right)
$$

and II is the second fundamental tensor

$$
\mathrm{II}=\left(\begin{array}{ll}
\frac{\partial \mathbf{n}}{\partial \mathbf{u}} \cdot \mathbf{u} & \frac{\partial \mathbf{n}}{\partial \mathbf{v}} \cdot \mathbf{u} \\
\frac{\partial \mathbf{n}}{\partial \mathbf{u}} \cdot \mathbf{v} & \frac{\partial \mathbf{n}}{\partial \mathbf{v}} \cdot \mathbf{v}
\end{array}\right)
$$

, and $(\mathbf{u}, \mathbf{v})$ are the axes of an arbitrary coordinate system in the tangent frame. We take $\mathbf{u}=\left(1,0,-n_{x} / n_{z}\right)^{T}$ and $\mathbf{v}=\left(0,1,-n_{y} / n_{z}\right)^{T}$ such that $\mathbf{u} \cdot \mathbf{n}=0$ and $\mathbf{v} \cdot \mathbf{n}=0$. The derivates of the normal are approximated using centered finite differences which is second-order accurate:

$$
\left(\frac{\partial \mathbf{n}}{\partial \mathbf{u}}\right)_{i, j}=\frac{\mathbf{n}_{i+1, j}-\mathbf{n}_{i-1, j}}{2}, \quad\left(\frac{\partial \mathbf{n}}{\partial \mathbf{v}}\right)_{i, j}=\frac{\mathbf{n}_{i, j+1}-\mathbf{n}_{i, j}}{2}
$$

\section{Results}

In our experiments, a HP ScanJet G4050 was used at a 2400 dpi and images were saved in RGB mode. Although HP ScanJet G4050 offers six-color scanning by two light sources which is employed in [8], we perform the scans in three-color mode by one light source. The intensity image for reconstruction was computed as $I=0.299 R+0.587 G+0.114 B$.

We tested the algorithm on a coin and a seal shown in Figure 1. Figure 4 and 5 show the acquired normal maps and mean curvature maps, respectively. The normal maps are displayed as RGB images where the RGB components correspond to the $n_{x}, n_{y}$ and $n_{z}$ values of the surface normal, respectively. In our implementation, $\mathrm{n}_{\mathrm{x}}$ maps from $(-1.0,1.0)$ to red $(0,255), \mathrm{n}_{\mathrm{y}}$ maps from $(-1.0,1.0)$ to green $(0,255)$ and $n_{z}$ maps from $(0.0,1.0)$ to blue $(0,255)$. The curvature maps are displayed as RGB images where positive curvatures correspond to red and negative to blue. The absolute values of curvatures are mapped to saturation in HSV color system. As seen from the figures, normal maps reveal fine surface detail that is difficult to see in color images.
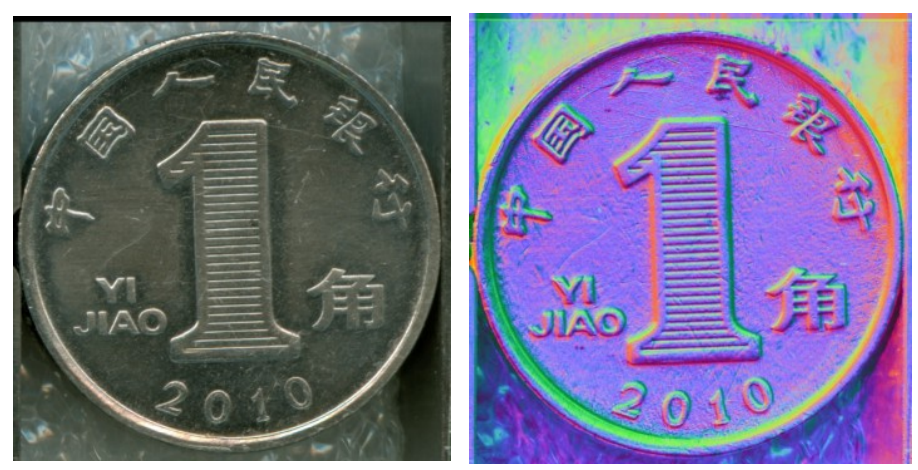

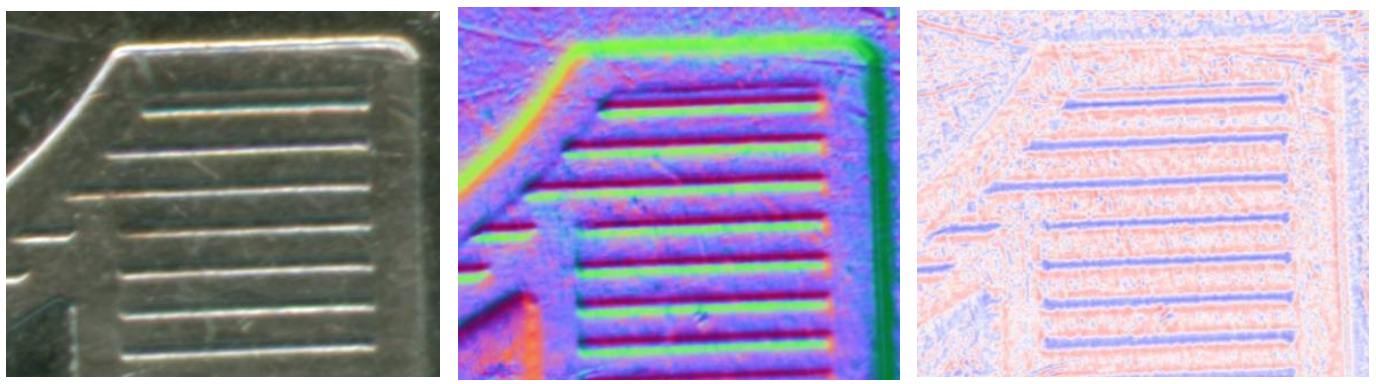

Figure 4: From left to right: color image, normal map and mean curvature map of a coin
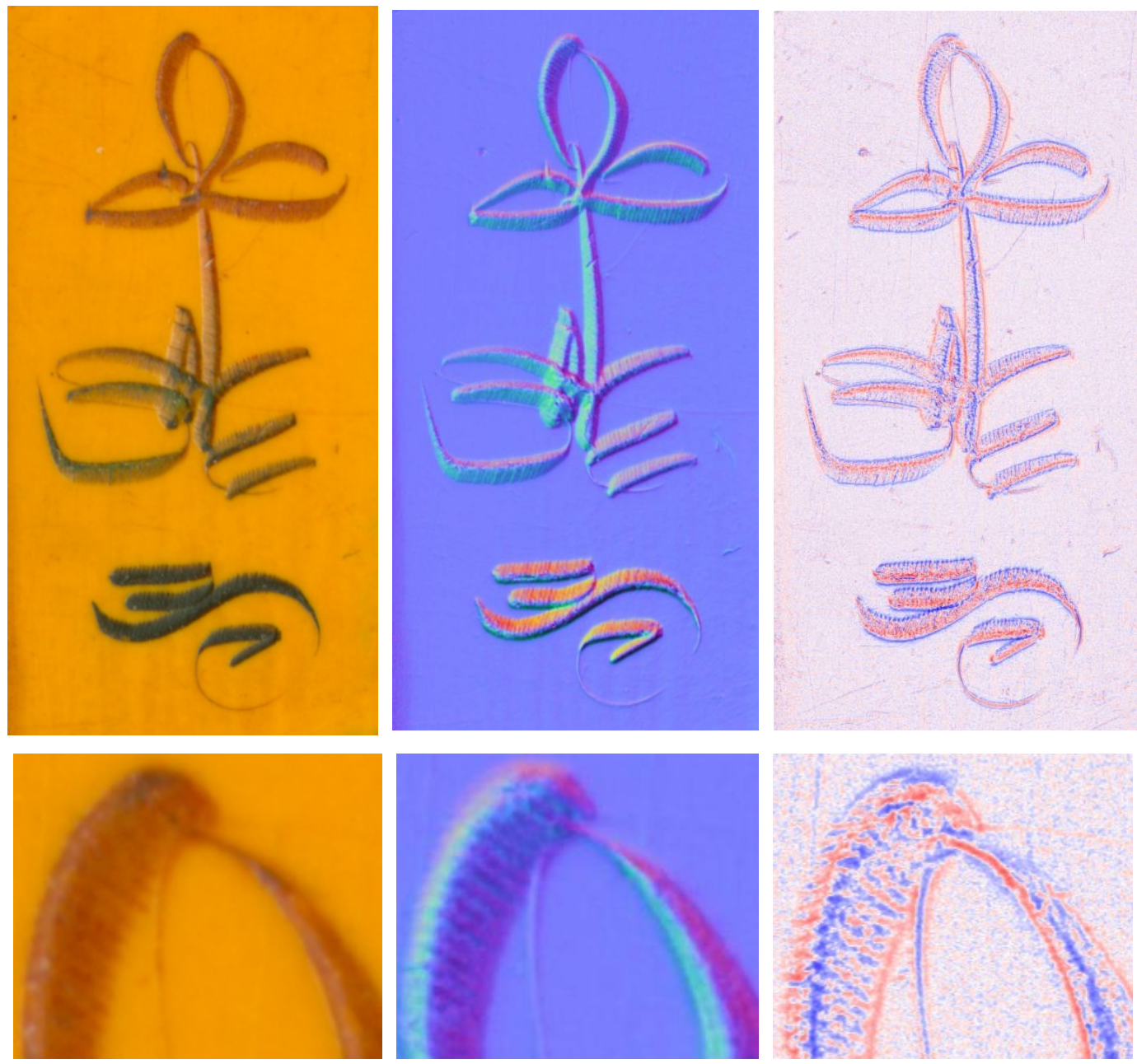

Figure 5: From left to right: color image, normal map and mean curvature map of a sculpture.

To evaluate the accuracy of our method, we make a check on the integrability constraint which boils down to check the mixed second partials are equal, $\frac{\partial^{2} z}{\partial x \partial y}=\frac{\partial^{2} z}{\partial y \partial x}$. So we expect that $\frac{\partial\left(n_{x} / n_{z}\right)}{\partial y}-\frac{\partial\left(n_{y} / n_{z}\right)}{\partial x}$ should be small at each point. The partial derivatives are estimated numerically and the differences between them are shown in Figure 6. Since most parts of the sculpture are flat, the difference between partial derivatives is small except at the engraved parts. 


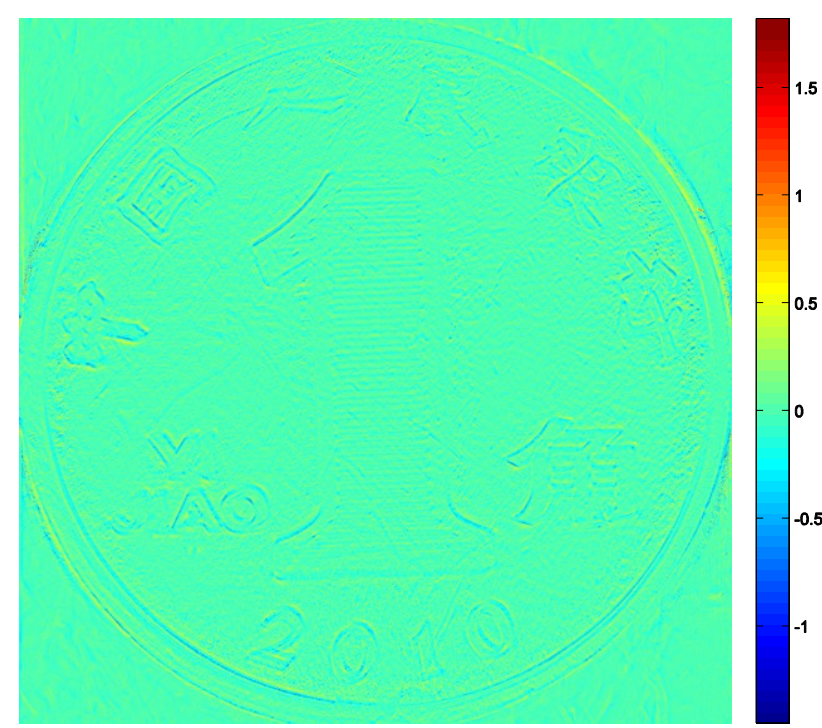

(a) Difference between partial derivatives for a coin

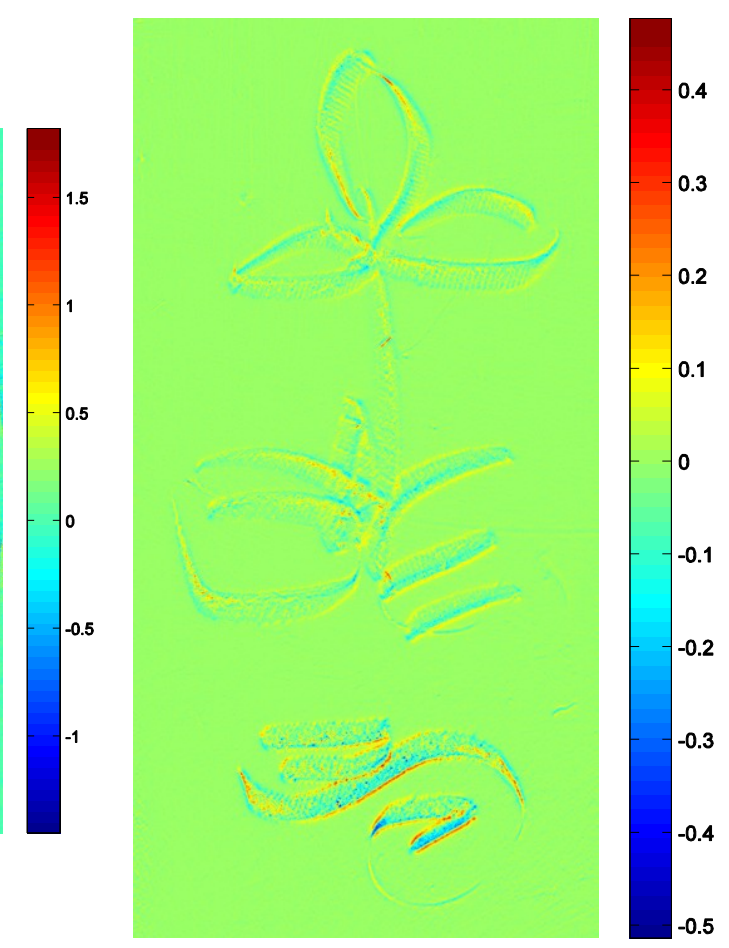

(b) Difference between partial derivatives for a sculpture

Figure 6: Validations of the integrability constraint

We also compute the residual error in the least-squares solution of Eq. (4), which provides a check on our measurement as well. Figure 7 shows the RMS residual errors for the coin and the sculpture. The average RMS error is 10.1 for the coin and 1.2 for the sculpture.

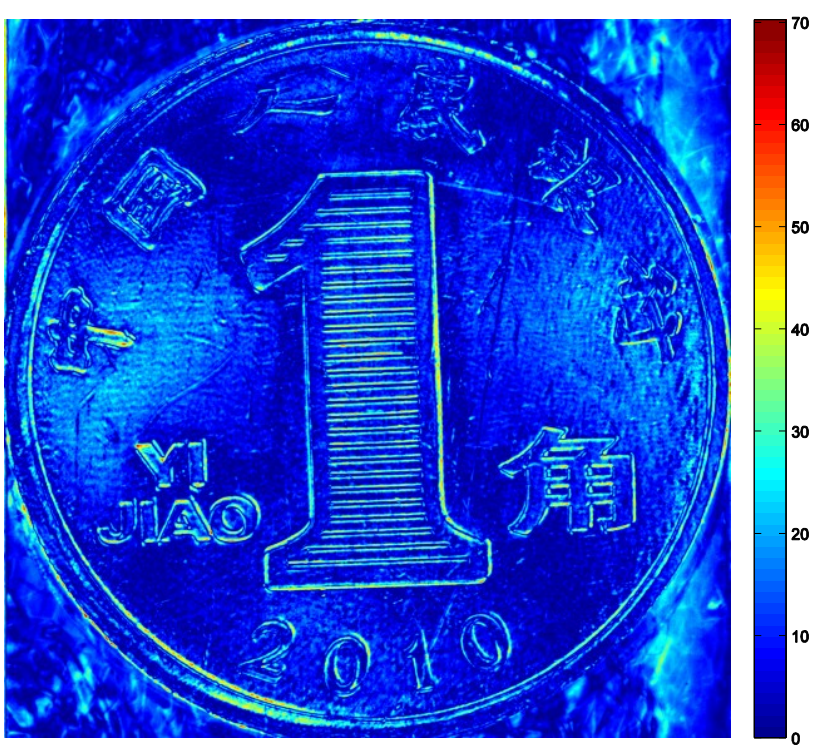

(a) a coin

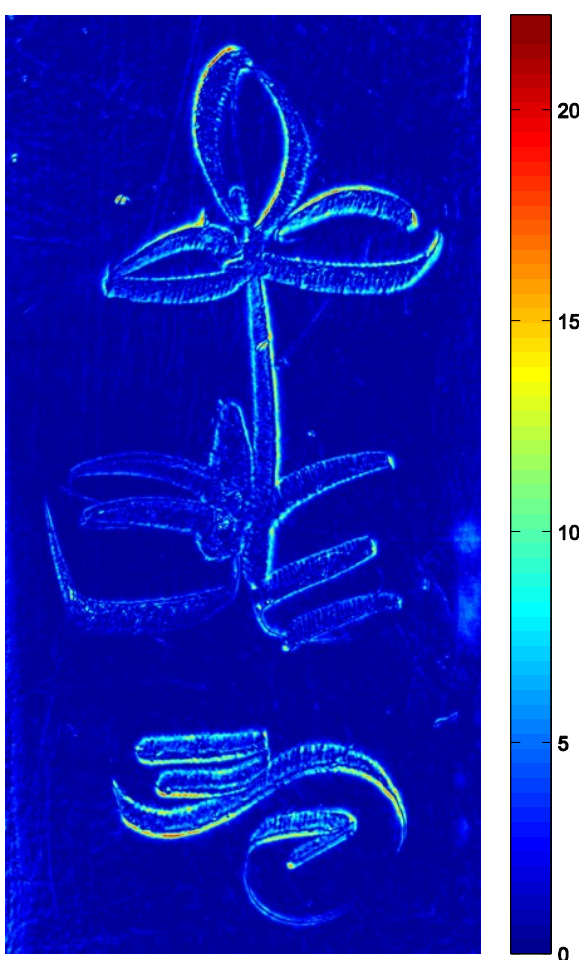

(b) a sculpture

Figure 7: RMS residual error

Our algorithm is implemented in $\mathrm{C}++$ language and the experiments were made on a 
notebook with $1.6 \mathrm{GHz}$ Intel Core Q720 processor and 3GB RAM memory. It takes about 10 seconds to extract the normal map and mean curvature map for images of $9 \mathrm{M}$ pixels.

\section{Conclusion}

In this paper we presented a method of acquiring normal maps of nearly flat objects with a flatbed scanner. The method computes surface normals from four scans with different object rotations based on the principle of photometric stereo. By exploiting the flatbed scanner's high resolution and the simple setup, the approach is applicable to small objects having a nearly flat surface such as in archaeological domain. As stated in [8], the limitation of our technique is due to the low depth-of-field of the scanner and the depth of geometry we can handle is approximately a few millimeters.

In the future the proposed technique will be considered for depth reconstruction of historical artifacts by integrating the normals.

\section{Acknowledgments}

Authors are grateful to colleagues for constructive comments and suggestions, to anonymous reviewers for their constructive comments and hints that helped to improve the manuscript significantly.

This work was supported by China-Czech Scientific and Technological Collaboration Project (40-8), Shandong Natural Science Foundation of China (Grant No. ZR2010FM046) and Project of Ministry of Education of the Czech Republic (project LH12181).

\section{References}

[1] Micah K. Johnson, Forrester Cole, Alvin Raj, and Edward H. Adelson. Microgeometry capture using an elastomeric sensor. ACM Trans. Graph. 30, 4, Article 46 (July 2011), 8 pages.

[2] Micah K. Johnson, and Edward H. Adelson. Retrographic sensing for the measurement of surface texture and shape. In Proceedings of the IEEE Computer Society Conference on Computer Vision and Pattern Recognition, 2009,1070--1077.

[3] B. Brown, C. Toler-Franklin, D. Nehab, M. Burns, D. Dobkin, A. Vlachopoulos, C. Doumas, S. Rusinkiewicz, and T. Weyric. A system for high-volume acquisition and matching of fresco fragments: Reassembling Theran wallpaintings. ACM Trans. Graph., 27(3):84:1-9, 2008.

[4] Corey Toler-Franklin, Adam Finkelstein, and Szymon Rusinkiewicz. Illustration of complex real-world objects using images with normals. In Proceedings of the 5th international symposium on Non-photorealistic animation and rendering (NPAR '07). ACM, New York, NY, USA, 111-119, 2007.

[5] J. F. Blinn. Simulation of wrinkled surfaces. SIGGRAPH Comput. Graph., vol. 12, no. 3, pp. 286-292, 1978.

[6] Tai-Pang Wu, Chi-Keung Tang, Michael S. Brown, and Heung-Yeung Shum. 2007. ShapePalettes: interactive normal transfer via sketching. ACM Trans. Graph. 26, 3, Article 44 (July 2007).

[7] Robert J. Woodham. Photometric stereo: A reflectance map technique for determining surface orientation from image intensity. In Proc. 22nd SPIE Annual Technical Symposium, vol. 155, 1978, pp. 136-143.

[8] Ruggero Pintus, Thomas Malzbender, Oliver Wang, Ruth Bergman, Hila Nachlieli, Gitit Ruckenstein. Photo Repair and 3D Structure from Flatbed Scanners Using 4- and 2-Source Photometric Stereo. Computer Vision, 
Imaging and Computer Graphics. Theory and Applications, Springer Berlin Heidelberg, Volume 68, page 326-342, 2010

[9] William Clarkson, Tim Weyrich, Adam Finkelstein, Nadia Heninger, J. Alex Halderman, and Edward W. Felten. Finger printing Blank Paper Using Commodity Scanners. In Proceedings of the 2009 30th IEEE Symposium on Security and Privacy (SP '09). IEEE Computer Society, Washington, DC, USA, 301-314. 2009.

[10] Robert J. Woodham. Photometric Method for Determining Surface Orientation from Multiple Images. Optical Engineering, 19(1):139-144, 1980.

[11] Gary Bradski, Adrian Kaehler. Learning OpenCV: Computer Vision with the OpenCV Library. O'Reilly Media; 1st edition (October 1, 2008)

[12] Andriy Myronenko, Xubo B. Song. On the closed-form solution of the rotation matrix arising in computer vision problems. CoRR abs/0904.1613, 2009 